\title{
Realistic scenarios of missing taxa in phylogenetic comparative methods and their effects on model selection and parameter estimation
}

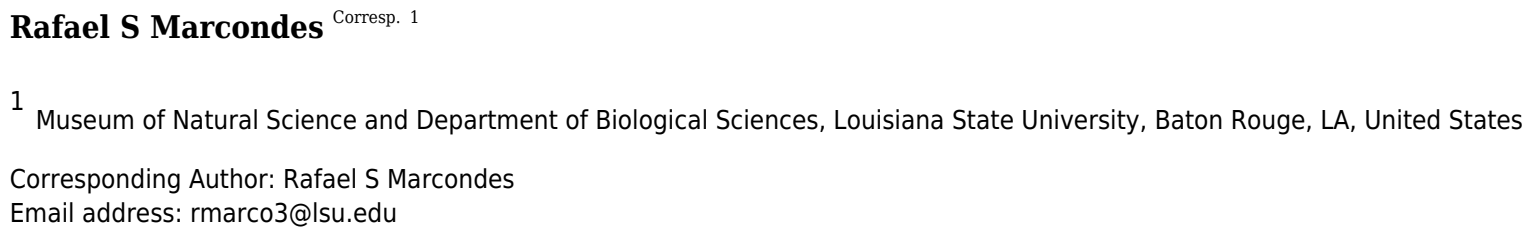

Model-based analyses of continuous trait evolution enable rich evolutionary insight. These analyses require a phylogenetic tree and a vector of trait values for the tree's terminal taxa, but rarely do a tree and dataset include all taxa within a clade. Because the probability that a taxon is included in a dataset depends on ecological traits that have phylogenetic signal, missing taxa in real datasets should be expected to be phylogenetically clumped or correlated to the modelled trait. I examined whether those types of missing taxa represent a problem for model selection and parameter estimation. I simulated univariate traits under a suite of Brownian Motion and Ornstein-Uhlenbeck models, and assessed the performance of model selection and parameter estimation under absent, random, clumped or correlated missing taxa. I found that those analyses perform well under almost all scenarios, including situations with very sparsely sampled phylogenies. The only notable biases I detected were in parameter estimation under a very high percentage (90\%) of correlated missing taxa. My results offer a degree of reassurance for studies of continuous trait evolution with missing taxa, but the problem of missing taxa in phylogenetic comparative methods still demands much further investigation. The framework I have described here might provide a starting point for future work. 
1 Realistic scenarios of missing taxa in phylogenetic comparative methods and their

2 effects on model selection and parameter estimation

3

4 Rafael S. Marcondes

5

6 Louisiana State University Museum of Natural Science and Department of Biological Sciences

7 Baton Rouge, LA 70802, USA

8

9 rmarco3@lsu.edu

10

11

12

13

14

15

16

17

18

19

20

21

22

23

24

25

26

27

28

29

30

31

32 


\section{Abstract}

Model-based analyses of continuous trait evolution enable rich evolutionary

insight. These analyses require a phylogenetic tree and a vector of trait values for the tree's terminal taxa, but rarely do a tree and dataset include all taxa within a clade.

37 Because the probability that a taxon is included in a dataset depends on ecological traits that have phylogenetic signal, missing taxa in real datasets should be expected to be phylogenetically clumped or correlated to the modelled trait. I examined whether those types of missing taxa represent a problem for model selection and parameter estimation.

41 I simulated univariate traits under a suite of Brownian Motion and Ornstein-Uhlenbeck models, and assessed the performance of model selection and parameter estimation under absent, random, clumped or correlated missing taxa. I found that those analyses perform well under almost all scenarios, including situations with very sparsely sampled phylogenies. The only notable biases I detected were in parameter estimation under a very high percentage (90\%) of correlated missing taxa. My results offer a degree of reassurance for studies of continuous trait evolution with missing taxa, but the problem of missing taxa in phylogenetic comparative methods still demands much further investigation. The framework I have described here might provide a starting point for

50 future work. 
56

57

\section{Introduction}

Phylogenetic comparative biology is a thriving field that uses phylogenies towards the goal of elucidating the historical mechanisms that have given rise to species and their traits. At the core of phylogenetic comparative methods are statistical models that translate ideas about evolutionary processes into the language of mathematics, thus allowing biological explanations to be quantitatively weighed against one another, and their implications to be explored in detail (Butler and King 2004, Cressler et al. 2015, Brown and Thomson 2018, Zuk and Travisano 2018). Model-based phylogenetic studies of univariate continuous trait evolution require at least two inputs: a phylogenetic tree of the species under study, and a vector of trait values for those species. One or more models are then typically fitted to the trait vector, conditioned on the phylogeny. The fits of these models can then be compared (often using Akaike information criteria) to assess which one offers the best explanation of the data. In addition, parameter estimates from fitted models can give insights into features of the evolutionary process that generated the data, such as adaptive peaks, rates of evolution and strength of selection (Beaulieu et al. 2012, O'Meara and Beaulieu 2014).

Phylogenies used in comparative studies are typically estimated from molecular sequences and trait data are typically generated from specimens deposited in natural history collections or, less often, from observations of live organisms. Because of differences in ecological characters such as range size, habitat preference, life history and behavior, not all taxa are equally likely to be available for inclusion in a molecular tree or trait dataset (Garamszegi and Møller 2011). Therefore, comparative studies often have missing taxa, that is, taxa that are members of the clade under study, but which 
79 researchers have been unable to include in their analyses because they were either not

80 included in the phylogeny or not accessible for measurement of traits (Thomson and

81 Shaffer 2010, Slater at al. 2012, Reddy 2014, Rabosky 2015). This usually results in the

82 missing taxa being excluded from the analyses, which has the potential to introduce

83 biases in model selection and parameter estimation (Garamszegi and Møller 2011,

84 Pennell et al. 2016).

85

Missing data have received significant attention in the context of nucleotide sequences used in phylogeny estimation (e.g., Wiens et al 2011, Jiang et al 2014, Eaton et al 2017). In stark contrast, missing data have received little attention in phylogenetic comparative biology (but see Garamszegi and Møller 2011), and, in the rare occasions when researchers assessed impacts of missing taxa in comparative models, they were simulated in a random fashion with respect both to trait values and to phylogeny (e.g., Ingram and Mahler 2013). In the next few paragraphs, I argue that missing taxa are unlikely to occur randomly and describe more realistic scenarios where missing taxa are phylogenetically clumped and/or correlated to the trait of interest. Next, I describe a set of simulations where my aims were two-fold: first, to conduct an initial, exploratory investigation of the impacts of realistic missing taxa on a limited set of models of univariate continuous trait evolution; and, second, by presenting a basic framework that can be taken up by other investigators, to instigate more attention and future research on this neglected issue in phylogenetic comparative biology. 
The multifarious ecological traits that influence the probability that a taxon is

102 sampled have phylogenetic signal. Consequently, missing taxa should be expected to be

103 phylogenetically clumped. The most important ecological trait influencing sampling

104 probability is rarity, broadly understood as the character of a taxon that has a low

105 abundance and/or a small geographical distribution (Gaston 1994). It is intuitive that rarer

106 taxa are more difficult to detect, observe, capture and collect, and thus more likely to be

107 missing from datasets than common taxa. Both components of rarity, abundance and

108 range size, have been shown to have phylogenetic signal, i.e., they are heritable at a

109 macroevolutionary level. For example, $41 \%$ of the variation in population density of North

110 American birds can be attributed to their taxonomic family (Maurer et al. 1991), and

111 taxonomic affiliation also explains variation in abundance among Neotropical rainforest

112 mammals (Arita et al 1990). As for range size, it has been shown to be heritable in studies

113 of mammals (DeSantis et al. 2012), birds (Waldron 2007, Herrera-Alsina and Villegas-

114 Patraca 2014), mollusks (Jablonski 1987) and herbaceous plants (Qian and Ricklefs

115 2004). That rarity has phylogenetic signal was also indicated by Fritz and Purvis' (2010)

116 finding that threatened species of British birds and of the world's mammals tended to be

117 phylogenetically clumped.

118 Beyond rarity, various other axes of an organism's ecological niche can affect its

119 probability of being sampled in comparative studies, and the pervasiveness of

120 phylogenetic niche conservatism (Losos 2008, Wiens et al. 2010, Crisp and Cook 2012)

121 is evidence of the high phylogenetic signal of ecological niches. Because taxa inhabiting

122 climates that are hostile to humans (for example, boreal organisms; Malaney and Cook

123 2018) are less likely to be sampled, climate is a particularly important niche axis in this 
124 context, and it has been repeatedly shown to display phylogenetic signal (reviewed by

125 Wiens and Graham 2005). Another important niche axis, habitat preference, also has

126 phylogenetic signal (Barr and Scott 2014) and is likely to affect sampling probability,

127 because forest-based organisms are more difficult to detect and collect than nonforest 128 organisms.

129 These taxonomic biases in rarity presumably translate into taxonomic biases in 130 availability of specimens and genetic samples. For example, Malaney and Cook (2018)

131 reported strong taxonomic imbalance in collections of North American mammals, with

132 Rodentia being comparatively overrepresented in relation to all other mammal orders,

133 and Reddy (2014) described similar imbalances for the availability of genetic data for the 134 world's birds.

135 In addition to being phylogenetically clumped, missing taxa might sometimes be 136 directly correlated to the trait under study, when taxa with a higher (or lower) trait value 137 have a lower probability of being sampled. For example, because taxa with small range 138 sizes are more difficult to sample, they will be missing from molecular datasets more often 139 than taxa with large ranges (Reddy 2014). This means that unsampled taxa will have a 140 smaller average range size than sampled taxa. Therefore, if we were undertaking a 141 comparative study of range size with incomplete taxon sampling, the distribution of trait 142 values in our sample will differ from the real distribution, potentially biasing model-based 143 analyses. This should also be the case for other ecological traits tightly linked to rarity 144 and sampling probabilities, such as abundance and some niche axes.

145 In sum, there is ample evidence of phylogenetic signal in ecological traits likely to 146 influence sampling probability for comparative studies. Consequently, sampling 
147 probability itself should display phylogenetic signal, and missing taxa are likely to be

148 phylogenetically clumped. There is also reason to expect some types of traits to be

149 directly correlated to sampling probabilities. Here, I present simulation-based analyses

150 examining how those realistic scenarios of missing taxa might affect the performance of 151 models of continuous trait evolution.

\section{Methods}

\section{Overview}

My simulations were designed to examine how realistic scenarios of missing taxa

affect model selection and parameter estimates for a single continuous trait in whose evolution we are interested, hereafter referred to as $\mathbf{T}$ (Table 1). I simulated $\mathbf{T}$ under a number of different models and then pruned $10 \%, 50 \%$ or $90 \%$ of terminal taxa from the tree under three different schemes (Figure 1): (1) randomly (rMT); (2) phylogenetically clumped missing taxa (cluMT); and (3) correlated to T (corMT). For comparison, I also ran simulations with no missing taxa (nMT). The sampling status of a taxon can be thought

162 of as a binary character, hereafter referred to as $\mathbf{S}$ (Table 1), with states 0 (missing) and

1631 (sampled). Once $\mathbf{T}$ and $\mathbf{S}$ were simulated, I pruned tips from the tree and dataset based

164 on $\mathbf{S}$ and, finally, I fitted all models to $\mathbf{T}$ and assessed support for the generating model, 165 as well as precision and bias of parameter estimates. I repeated the simulation 1000 times

166 for each combination of type of missing taxa, percentage of missing taxa, and model of T 167 trait evolution.

168 Before presenting the details of my simulations, I reiterate that I did not seek to 169 exhaust every possible scenario of missing taxa in phylogenetic comparative biology. 
170 Rather, I sought to explore the implications of two very specific scenarios that I argue are

171 likely in real datasets. There undoubtedly exist other possible, if less probable,

172 configurations of missing taxa, and they may have different impacts on model

173 performance, but addressing those was beyond the scope of my study. I also

174 acknowledge that, even within the scenarios I studied, some of my simulation settings

175 necessarily entailed some degree of arbitrariness, for example in the size of the trees or

176 in the parameter values I used to simulate various traits. Different choices might have led

177 to different results, but while exploring those choices would certainly be productive, it was

178 not my present objective in this initial, exploratory study.

\section{Details}

I started each simulation by generating a random phylogenetic tree under a pure-

182 birth model using the function rphylo in the R package ape (Paradis and Schliep 2019)

183 and rescaling the tree to unit height using the function rescale in the $\mathrm{R}$ package geiger

184 (Pennell et al. 2014). The size of each initial tree was set so that the number of tips after

185 dropping missing taxa was always equal to 300 . For example, when simulating $10 \%$

186 missing taxa, the initial number of tips was 333 , and when simulating $50 \%$ missing taxa it

187 was 600 . This ensured that my results were affected only by missing taxa per se, and not

188 by tree size, which is known to affect the performance of models of trait evolution

189 (Beaulieu et al. 2012, Boettiger et al. 2012).

190 To represent regimes underlying variation in BMS and OUM parameters (see

191 below), I simulated the evolution of a binary trait $\mathbf{R}$ under an equal rates MK model with

192 a transition rate $q=0.5$ (Table 1 , Figure $1 \mathrm{~d}$ ), and I ensured that the smallest of the two 
193 regimes always included no fewer than $25 \%$ and no more than $45 \%$ of tips after dropping

194 the tips representing missing taxa. To simulate this trait, I used the function

195 simulate_mk_model in the R package castor (Louca and Doubeli 2017).

196 After simulating the tree and $\mathbf{R}$, I simulated $\mathbf{T}$ (Table 1), the continuous trait of

197 interest, under one of four models using the function OUwie.sim in the R package OUwie

198 (Beaulieu et al. 2012): single-rate Brownian Motion (BM), single-optimum Ornstein-

199 Uhlenbeck (OU), multiple-rate Brownian Motion (BMS), and multiple-optimum Ornstein-

200 Uhlenbeck (OUM). Under BM, changes in trait value are purely nondirectional and

201 governed by a single parameter, $\sigma^{2}$ (sigma-square), that determines the rate of evolution

202 (Felsenstein 1985). Under OU, trait evolution is controlled by a nondirectional component

203 represented by $\sigma^{2}$ as well as by a directional component under which trait values change

204 preferentially towards an optimum ( $\theta$, theta) with strength of attraction $\alpha$ (alpha) (Hansen

205 1997). BMS and OUM represent variations of $B M$ and $O U$ in which $\sigma^{2}$ and $\theta$, respectively,

206 are allowed to assume different values depending on regimes (trait $\mathbf{R}$ in my simulations)

207 reconstructed a priori on the phylogeny (Butler and King 2004, O'Meara et al 2006,

208 Beaulieu et al 2012).

209 For each model, I set the rate parameter $\sigma^{2}$ at the root $\left(\sigma^{2}\right)$ to a value of 0.5 , and

210 only for the BMS model it shifted to $\sigma^{2}{ }_{1}=1$ in the derived regime. For the OU and OUM

211 models, I set the optimum parameter $\theta$ to 10 at the root $\left(\theta_{0}\right)$, with a shift to $\theta_{1}=11$ in the

212 derived regime under OUM. The a parameter of OU and OUM models, representing the

213 strength of attraction to the optimum, was always constant at 1.5. This a value

214 corresponds to a phylogenetic half-life (the time, as a proportion of the tree height, that a 
215 trait takes to evolve halfway towards the adaptive peak $\theta$ ) of 0.46 , thus representing an

216 OU process of moderate strength.

217 For the cluMT scenario (Figure 1B), I simulated $\mathbf{S}$, the trait determining the

218 sampling status of each tip (Table 1), under a threshold model (Felsenstein 2005, Fritz

219 and Purvis 2010), where $\mathbf{S}$ is underlain by a continuous liability trait $\mathbf{L}$ (Table 1 ). The state

220 of $\mathbf{S}$ for each tip depends exclusively on whether $\mathbf{L}$ is above or below a certain threshold

221 value. Because sampling status is likely to be a highly complex trait determined by

222 innumerable neutral and adaptive evolutionary forces, its evolution should resemble a

223 purely nondirectional process over macroevolutionary time and be best described by a

224 simple Brownian Motion model (O'Meara et al. 2006), which I thus chose to simulate L. I

225 used a $\sigma^{2}$ value of 1 in the function OUwie. sim. The threshold for $\mathbf{S}$ was set based on the

226 desired percentage of missing taxa, so that, for instance, when simulating $10 \%$ missing

227 taxa, the tips with the $10 \%$ lowest values of $L$ were assigned state 0 and the tips with the $22890 \%$ highest values were assigned state 1.

229 For corMT (Figure 1C), I simulated $\mathbf{S}$ non-phylogenetically, using the $\mathrm{R}$ native

230 function sample.int to sample tips to be dropped. I provided that function with an integer

231 corresponding to the total number of tree tips (argument $n$ ), the desired number of tips to

232 be dropped (size), and a vector of weights for obtaining the elements of the vector being

233 sampled (prob). Those weights were calculated, for each tip, as:

234

235

$$
w=\frac{\mathrm{t}}{\operatorname{sum}(\mathrm{T})}-\frac{\min (\mathrm{T})}{\operatorname{sum}(\mathrm{T})}
$$


237 ,where $t$ is the value of the trait of interest for that tip, and $T$ is the vector of $t$ values for all

238 tips. Sample.int then used those weights to sample size integers from the interval 1:n.

239 The sampled integers correspond to tips to drop. This procedure results in the sampling

240 probability of each tip being linearly proportional to its $\mathbf{T}$ value. The tip with the lowest $\mathbf{T}$

241 value had a $100 \%$ chance of being sampled, and tips with a higher $\mathbf{T}$ value were

242 probabilistically more likely, but not certain, to be missing (Figure 1C).

243 Due to the different ways in which missing tips were sampled, in the cluMT and

244 corMT scenarios the number of missing tips always corresponded exactly to the desired

245 proportion, but in the rMT case, that number varied slightly due to the probabilistic

246 sampling. For example, under 50\% rMT the actual proportion of missing tips varied from

2470.43 to 0.57 . However, this does not bias my inferences because over 1000 simulations

248 the number of missing tips will average to the desired proportion, and the results will

249 reflect that.

250 For each simulation in each scenario of missing taxa, I quantified the phylogenetic

251 signal in S using Fritz and Purvis' (2010) D statistic, calculated with the function phylo.d

252 in caper (Orme et al. 2017). D equals 0 when a binary trait has evolved under a threshold

253 model with a Brownian liability as described above, and 1 when it has a phylogenetically

254 random distribution at the tips of the tree.

255 Once $\mathbf{T}$ had been simulated and tips had been pruned based on $\mathbf{S}$, I used OUwie

256 to fit each of the four models to $\mathbf{T}$ and assess their support using sample size-corrected

257 Akaike information criteria (AICc; Burham and Anderson 1998), under the expectation

258 that the generating model should have the lowest AICc score. For each combination of

259 generating model, scenario of missing taxa, and percentage of missing taxa, I also 
260 calculated the median delta AICc of the generating model in the set of simulations in

261 which it was not the top model. Delta AICc equals the AICc score of the focus model

262 minus the AICc score of the model with the most support (lowest AICc), and is thus a

263 measure of relative support of a model compared to the top model.

264 Finally, I computed the bias and precision of parameter estimates under each

265 scenario to assess how they were affected by missing taxa. I calculated bias as the mean

266 parameter estimate minus the generating parameter value. I calculated precision as the

267 median absolute deviation (median deviation from the median) of parameter estimates

268 among simulations. I used this statistic in lieu of the simple variance because it is more

269 robust to outliers. I normalized both bias and precision by dividing them by the generating

270 parameter values.

271

272

\section{Results}

273

The mean value of Fritz and Purvis' $D$ statistic for $\mathbf{S}$, the trait determining the sampling status of each tip, was 1.004 and -0.019 across all simulations in the rMT and 275 cluMT scenarios respectively, indicating, as intended, low phylogenetic signal of missing taxa in the former, and high phylogenetic signal of missing taxa in the latter. For corMT,

277 because $\mathbf{S}$ was correlated to $\mathbf{T}$, its phylogenetic signal was computed separately 278 depending on T's model of evolution. The mean value of Fritz and Purvis' D statistic in 279 that scenario was $0.921,0.931,0.944$, and 0.932 for $\mathrm{T}$ simulated under BM, BMS, OU 280 and OUM, respectively.

281 Clumped and correlated missing taxa resulted at most in a slight increase in the 282 model selection error rate compared to no missing taxa or to random missing taxa, as 
283 indicated by the number of simulations in which the generating model did not have the

284 lowest AICc among the four models (Table 2, Figure 2). For example, when the

285 generating model was OUM, it failed to receive the most support in 22 out of 1000

286 simulations under a no missing taxa scenario, 4 out of 1000 under $90 \%$ rMT, 24 out of

2871000 under $90 \%$ cluMT, and 11 out of 1000 under $90 \%$ corMT. The model selection error

288 rate did not consistently increase with the percentage of missing taxa under any scenario,

289 but under all scenarios the error rate was higher (always $\sim 30 \%$ ) when BM was the

290 generating model, usually followed by OU, then by BMS and OUM (Table 2, Figure 2).

291 BM was most often confused for BMS and OU, and more rarely confused for OUM (Figure

292 2). Likewise, BMS was confused more often for BM and OU than for OUM (Figure 2). In

293 contrast, OU was often confused for OUM. Finally, OUM was only very rarely not selected

294 as the top model when it was the generating model (Table 2, Figure 2), indicating that it

295 leaves the strongest signature on the data among all models examined here.

296 Further revealing that missing taxa have little impact on model selection, the

297 median delta AICc of the generating model in the set of simulations where it was not the

298 top model was almost always $\leq 2$ (Table 3 ). This means, following Burham and Anderson's

299 (1998) rule of thumb that "models having delta AICc $\leq 2$ have substantial support", that the

300 generating model still had relatively high support even in the cases where it was not the 301 top model.

302 I also examined the bias (Table 4) and precision (Table 5) of parameter estimates

303 across simulations in each scenario. Because I normalized these metrics, they are directly

304 comparable across parameters. The absolute bias (Table 4) of $\sigma^{2}{ }_{0}$ was always low $305(<0.0374)$ for all models and almost always remained so for $\sigma^{2}{ }_{1}$ in BMS, even though $\sigma^{2}{ }_{1}$ 
306 covered much smaller proportions of my simulated trees. The absolute bias of $\theta_{0}$ also

307 remained fairly low $(<0.01)$, but the absolute bias of $\theta_{1}$ was noticeably higher across most

308 situations (often >0.2). Finally, $\alpha$ had by far the greatest bias of all parameters (almost

309 always $>0.1$ ).

310 Notably, although biases were generally low, they often tended to be systematic,

311 meaning they were always in the same direction. $\sigma^{2}$ was consistently underestimated in

312 BM and BMS models and overestimated in OU and OUM. a was always overestimated,

313 and $\theta_{1}$ always underestimated. Only for $\theta_{0}$ was bias not systematic, as it was sometimes

314 positive and sometimes negative. Regarding precision of parameter estimates, across all

315 scenarios it tended to be to be better (meaning parameter estimates were more

316 concentrated around the generating parameter value) for $\theta$, followed by $\sigma^{2}$, and worst for

317 a (Table 5).

318 Interestingly, looking at each parameter individually, both bias and precision were

319 largely similar across almost all scenarios, revealing that missing taxa had little effect on

320 parameter estimation. Only at $90 \%$ corMT did the bias of most parameters worsen relative

321 to other scenarios and percentages (Table 4, Figure 3). For example, the bias of $\sigma^{2}{ }_{0}$ in

322 the BM model was -0.0084 under nMT, -0.0067 under $90 \%$ rMT, -0.0012 under $90 \%$

323 cluMT, and jumped to -0.0374 under $90 \%$ corMT. Similarly, the bias of $\theta_{0}$ in the OUM

324 model was -0.0033 at $\mathrm{nMT},-0.0032$ at $90 \% \mathrm{rMT},-0.0036$ at $90 \%$ cluMT, and much greater

325 at -0.0142 at $90 \%$ corMT. In contrast, the precision of parameter estimates did not

326 deteriorate consistently even under $90 \%$ corMT (Table 5). For example, for $\sigma^{2}{ }_{0}$ in the BM

327 model, the precision was 0.0834 under $90 \%$ corMT compared to 0.0800 under nMT and

$328 \quad 0.0862$ under $10 \%$ corMT. 


\section{Discussion}

\section{Model performance in the presence of missing taxa}

Random and phylogenetically clumped missing taxa had little effect on model phylogenetic trees, even when $90 \%$ of taxa in a tree were missing. This result can be intuitively understood by appreciating that neither of these two scenarios change the distribution of trait values at the tips of the tree. Under random missing taxa, each tree tip has the same chance of being missing, independent of its trait value or phylogenetic position. Under phylogenetically clumped missing taxa, the chances of a tip being missing are independent of trait values, meaning that if a taxon is missing, it is likely that its close relatives will also be missing. These close relatives probably have similar trait values, but

341 the distribution of trait values at the tips will not change because clumps of missing taxa

342 will be evenly distributed across the tree, and trait values are not correlated across these

343 clumps (Figure 1). The only notable effect of missing taxa was when $90 \%$ of taxa were

344 missing under a scenario in which sampling probability was correlated to the trait of 345 interest (corMT). In that situation, there was a noticeable deterioration in the bias, but not

346 the precision, of all parameters. This result can be understood by realizing that this is the

347 only type of missing taxa that alters the distribution of trait values at the tips, thus 348 misleading estimates. 
Even though my main interest was exploring the implications of missing taxa, and

352 those proved to be minor, it is also valuable to discuss performance in a scenario without

353 missing taxa, seeing as model performance, in particular of OU models, has received

354 significant recent attention in the form of simulation studies (Beaulieu et al. 2012, Ho and

355 Ané 2014, Cooper et al. 2015, Cressler et al. 2015).

BM was by far the model that most often failed to be selected by AICc when it was

357 in fact the generating model, with an error rate of $\sim 30 \%$ across all scenarios (Table 2 ,

358 Figure 2). This suggests that BM is quite prone to mimicking patterns expected under

359 other models, echoing Slater et al.'s (2012b) finding, in a simulated birth-death phylogeny

360 with 100 extant tips, that statistical power is low to favor BM over other candidate models

361 even when it is the generating model. In particular, in my simulations with no missing taxa,

362 a single-peak OU model was mistakenly selected over BM in $12.2 \%$ of the simulations

363 (Figure 2) when BM was the generating model. This result can be compared to Cooper

364 et al.'s (2015), who similarly simulated BM datasets and computed how often an OU

365 model was mistakenly selected as the best-fitting model. However, instead of using

366 Akaike information criteria for model selection, they used likelihood-ratio tests and Bayes

367 factors. The error rate they found was considerably lower than what I found using AICc,

368 for example $5.5 \%$ and $0.2 \%$ in pure-birth trees with 200 tips using likelihood-ratio tests 369 and Bayes factors respectively.

370 To explore the discrepancy between mine and Cooper et al.'s (2015) results, I ran

371 likelihood ratio tests (LRTs) on the results of my nMT simulations under BM (details

372 presented as supplementary material). I found that LRTs incorrectly preferred OU over

373 the true BM model in $6.7 \%$ of the simulations, a number much lower than the AICc error 
374 rate of $12.2 \%$ and more in line with Cooper et al.'s (2015) $5.5 \%$. This result suggests that

375 LRTs may perform better than AICc as a tool to discriminate between BM and OU models

376 in comparative datasets, an issue that, while beyond the purview of the present paper,

377 deserves further investigation.

378 Regarding parameter estimates, $\alpha$ was the hardest parameter to estimate in every

379 scenario, seeing as it had the largest biases and poorest precisions, sometimes by orders

380 of magnitude, compared to all other parameters (Tables 4 and 5). This result is consistent

381 with Cressler et al.'s (2015), who found the related parameter $\eta$ (selection opportunity,

382 equaling the product of $\alpha$ and the height of the phylogeny) the hardest parameter to

383 estimate, and $\theta$ the easiest in OUM models. In my results $\alpha$ and $\sigma^{2}$ estimates tended to

384 have a consistent upward bias in OUM models, whereas $\theta_{1}$ was biased downwards and

$385 \theta_{0}$ did not have a consistent upwards or downwards bias (Figure 3 ). These results are

386 similar to Beaulieu et al.'s (2012), except, interestingly, for $\theta_{0}$, which Beaulieu et al. (2012)

387 found to be underestimated as well, although in that case simulations were based on

388 more complex models.

389

\section{Broader implications for models of trait evolution in the presence of missing}

391 taxa

392

My results bear on the issue of birth-death polytomy resolver (BDPR) algorithms,

393 such as those of Kuhn et al. (2011) and Thomas et al. (2013), that impute the position of

394 missing taxa onto a phylogeny based solely on taxonomic information and a

395 diversification model. Jetz et al. (2012) used a BDPR to add unsampled taxa to a species-

396 level phylogeny of the world's birds, amounting to $33 \%$ of all species in the final tree. This 
397 approach was shown by Rabosky (2015) to systematically and severely bias downstream

398 analyses of trait macroevolution. For example, when using a BDPR algorithm, Rabosky

399 (2015) found the Brownian rate parameter $\sigma^{2}$ to be overestimated by an average factor

400 of 3.51 times, compared to a bias of no more than 0.02 in any of my scenarios with missing

401 taxa (Table 4). Therefore, my results suggest that the reliability of BDPR algorithms may

402 be a moot point for studies concerned mainly with continuous traits, because model

403 selection and parameter estimation might perform better on phylogenies with a very high

404 proportion of realistic missing taxa than on BDPR-imputed phylogenies. This is in 405 accordance with Rabosky's (2015) "genes only" scenario, where he ran trait evolution

406 analyses with minimal bias after pruning BDPR-imputed species from the Jetz et al.

407 (2012) tree. However, this is not to say that BDPR algorithms are never useful. We need

408 to remember that BDPRs do demonstrably improve the performance of comparative

409 studies that do not include traits, such as speciation-extinction analyses (Rabosky 2015).

410 Even though my and Rabosky's (2015) findings offer some reassurance for the

411 use of incompletely sampled phylogenies for studies of continuous trait evolution, caution

412 must be taken with ecological traits that are correlated to sampling probabilities, a

413 scenario that caused biased estimates in my simulation. This includes traits tightly linked

414 to rarity, such as abundance and range size (Gaston 1994), as well as niche axes such

415 as habitat type. Rarity is an emergent property of species and not a property of individuals.

416 Traits with potential to bias model-based analyses will likely also be similarly emergent

417 species-level traits. However, it is difficult to generalize about the types of traits that will

418 negatively affect parameter estimates, because they will vary depending on the ecology

419 and life-history of the clade under study. Because ecology and life-history are most well- 
420 known by the researchers investigating specific clades, it is advisable that these

421 researchers use their biological intuition to stay mindful of traits that they believe may be

422 linked to rarity and sampling probabilities in their study organisms, especially in situations

423 with extremely high proportions of missing taxa.

424 The statistical power of a tree and comparative dataset to correctly identify the

425 model that generated the data and to estimate model parameters scales with tree size

426 (Beaulieu et al. 2012, Boetigger et al. 2012). For example, trees with up to 128 tips

427 sometimes do not allow the correct selection of an OUM model over OU (Beaulieu et al.

428 2012). The problem of low statistical power in small trees is related to, but distinct from,

429 the problem of missing taxa. Phylogenetic trees may have small sizes and consequent

430 low statistical power for three reasons: (1) because taxon sampling is complete but the

431 clade under study is in fact a small clade; (2) because the clade under study is known to

432 be larger but researchers were unable to sample many known taxa, or (3) because the

433 clade is larger but contains many undiscovered, undescribed or recently extinct species,

434 so that researchers may not even be aware that their study contains missing taxa. In my

435 simulations, I isolated the missing taxa problem from the tree size problem by keeping

436 the number of tips constant after pruning missing taxa, but this is not realistic. In practice

437 the two problems cannot be addressed separately, as under reason 2 above, and may

438 not even be distinguishable, as under reason 3 above. Assessment of statistical power

439 should be standard in comparative analyses. A simulation-based method to accomplish

440 this was described by Boetigger et al. (2012), but it implicitly assumes complete taxon

441 sampling (reason 1 above) and it is unclear if it is adequate in situations with missing

442 taxa. Slater et al. (2012a) described a method to account for missing taxa in comparative 
443 studies, but it assumes that missing taxa are phylogenetically random, a scenario that I

444 argue is unlikely. As far as I am aware, there are no available methods to account for

445 clumped, correlated or unknown (reason 3 above) missing or recently extinct taxa.

446 In fact, the scenarios produced by my simulations, where I pruned terminal

447 branches from a tree to represent missing taxa, are indistinguishable from scenarios that

448 would be produced by recent extinctions (i.e., extinction at the tips of the phylogeny only).

449 Therefore, my findings might also be interpreted in that context as meaning that random,

450 clumped or correlated recent extinction appear to have little effect on model performance.

451 My results should not, however, be extended to scenarios with extinction in internal

452 branches, which are likely to be prevalent in real datasets (Stadler 2010, Slater et al.

453 2012b, Stadler et al. 2018). As a case in point, Slater et al. (2012b) found the performance

454 of model selection to significantly deteriorate when randomly-simulated fossil (extinct)

455 taxa were pruned from trees. This deterioration is likely to be even greater if extinction is 456 clumped (Vamosi and Wilson 2008, Rabosky 2009) or correlated (FitzJohn 2010, Harvey 457 and Rabosky 2018) to trait values.

458 The roster of phylogenetic models of trait evolution is large and ever-growing, and 459 here I examined the effects of missing taxa on only a very small part of that universe. 460 Rabosky (2015) found that results for discrete traits were generally similar to those for 461 continuous traits, but before further analyses it is difficult to speculate on how my findings 462 might apply to other models. Furthermore, the scenarios I used to simulate missing taxa 463 were quite limited. Whereas it is clear that missing taxa will often be phylogenetically 464 clumped and/or correlated to the investigated traits, I cannot be sure that the ways in 465 which I simulated those scenarios are optimal. In particular, my non-phylogenetic 
466 simulation of corMT is perhaps less than ideal. A more realistic way to simulate that

467 scenario would be under a multivariate phylogenetic model where the value of a liability

$468 \mathrm{~L}$ is governed both by a Brownian Motion process and by a correlation with a trait of

469 interest $\mathbf{T}$, which may be evolving the same or a different model than $\mathbf{L}$. However, to the

470 best of my knowledge, that type of multivariate model is not currently available.

Another caveat is that, my simulations notwithstanding, scenarios of missing taxa

472 indisputably exist that do severely mislead phylogenetic comparative methods. For example, without delving into details, a situation in which all taxa at one extreme of the

474 trait value distribution are deterministically missing from a dataset (i.e., all tips with the $\mathrm{X} \%$ greatest values of $\mathbf{T}$ are always unsampled) would truncate the distribution of trait values, altering its mean and variance and likely leading to selection of incorrect models and poor performance of parameter estimates. However, that scenario is unrealistic, because even the rarest taxa will always have a non-zero chance of being discovered, collected and sampled.

As a final cautionary note, my study, as all simulation studies, is only valuable to 481 the extent that our models are accurate. In reality, evolution is likely to proceed in a much 482 more heterogenous manner than reflected in any of our current, simplistic models of trait 483 evolution. We do not know, and might never know, the real generating "models" of 484 empirical datasets. Moreover, actual data, unlike the data I simulated here, are not ideal 485 (e.g., they have measurement error). Therefore, I recommend that my results be not uncritically taken to be applicable whenever any of the models I tested here is fit to real data. The processes that generated a given real dataset may or may not be as robust to missing taxa as are our simple models. Rather, I expect my results to prompt empirical 
489 phylogenetic comparative biologists to think critically about missing taxa in their datasets

490 and how they might be distributed. The better the fit of a dataset to a model is, the more

491 likely it is that my findings on the effects of missing taxa will be applicable.

492

493

\section{Conclusion}

494

My results demonstrate that realistic scenarios of missing taxa do not affect the

performance of selected simple models of univariate continuous trait evolution, except for parameter estimates in situations with a very high proportion of correlated missing taxa. Understanding the impact of missing taxa in comparative methods is a multifaceted problem that still demands much additional work. Topics that are ripe for further study, considering my results, include the effect of missing taxa on models of discrete trait evolution, exploration of better ways to simulate correlated missing taxa, examinations of

501 the level of phylogenetic signal in missing taxa in real datasets, and disentangling the 502 problem of small tree size from the problem of missing taxa. The framework I have provided here will be a starting point for such studies. To facilitate that, $\mathrm{R}$ scripts to

504 replicate my simulations are available on GitHub. Proponents of existing and novel 505 models of trait evolution will benefit by using this framework to more explicitly and

506 thoroughly investigate model performance under realistic scenarios of missing taxa. 507

508

509

\section{Acknowledgments}

This study benefitted from feedback from Jeremy Brown, Robb Brumfield, Jake Esselstyn and all participants in the Phyleaux discussion group at LSU. All simulations were run in the Odyssey computing cluster at Harvard University, access to which is 
512 thanks to Scott Edwards. This research was supported in part by NSF grant DEB-

513 1146265, and by a "Science Without Borders" doctoral fellowship to RSM from Brazil's

514 National Council for Scientific and Technological Development (CNPq; 201234/2014-9).

515

516 Data and code archival statement

$517 \quad \mathrm{R}$ scripts to replicate the analyses and results of the simulations are available at

518 https://github.com/rafmarcondes/Missing_taxa.

519

520 References cited

521

522 Arita, H. T., J. G. Robinson, K. H. Redford, H. T. Arita, J. G. Robinson, and K. H.

523 Redford (1990). Rarity in Neotropical Forest Mammals and Its Ecological

524 Correlates. Conservation Biology 4:181-192.

525 Barr, W. A., and R. S. Scott (2014). Phylogenetic comparative methods complement

526 discriminant function analysis in ecomorphology. American Journal of Physical

527 Anthropology 153:663-674. doi: 10.1002/ajpa.22462

528 Beaulieu, J. M., D. C. Jhwueng, C. Boettiger, and B. C. O'Meara (2012). Modeling

529 stabilizing selection: Expanding the Ornstein-Uhlenbeck model of adaptive

530 evolution. Evolution 66:2369-2383. doi: 10.1111/j.1558-5646.2012.01619.x

531 Boettiger, C., G. Coop, and P. Ralph (2012). Is your phylogeny informative? Measuring

532 the power of comparative methods. Evolution 66:2240-2251. doi: 10.1111/j.1558-

$533 \quad 5646.2011 .01574 . x$

534 Brown, J. M., and R. C. Thomson (2018). Evaluating Model Performance in 
535 Evolutionary Biology. Annual Review of Ecology, Evolution, and Systematics

536 49:annurev-ecolsys-110617-062249. doi: 10.1146/annurev-ecolsys-110617-062249

537 Butler, M. A., and A. A. King (2004). Phylogenetic Comparative Analysis: A Modeling

538 Approach for Adaptive Evolution. The American Naturalist 164:683-695. doi:

$539 \quad 10.1086 / 426002$

540 Burham, K. P., and D. R. Anderson. (1998). Model Selection and Inference: A Practical

541 Information-Theoretuc Approach. Springer Verlag, New York.

542 Cressler, C. E., M. A. Butler, and A. A. King. (2015). Detecting adaptive evolution in

543 phylogenetic comparative analysis using the Ornstein-Uhlenbeck model.

$544 \quad$ Systematic Biology, 64(6), 953-968.

545 Crisp, M. D., and L. G. Cook (2012). Phylogenetic niche conservatism: What are the

546 underlying evolutionary and ecological causes? New Phytologist 196:681-694. doi:

$547 \quad$ 10.1111/j.1440-1827.1997.tb04503.x

548 DeSantis, L. R. G., R. A. Tracy, C. S. Koontz, J. C. Roseberry, and M. C. Velasco

549 (2012). Mammalian niche conservation through deep time. PLoS ONE 7. doi:

$550 \quad$ 10.1371/journal.pone.0035624

551 Eaton, D. A. R., E. L. Spriggs, B. Park, and M. J. Donoghue (2017). Misconceptions on

552 Missing Data in RAD-seq Phylogenetics with a Deep-scale Example from Flowering

$553 \quad$ Plants. Systematic Biology 66(3):399-412.

554 Felsenstein, J. (1985). Phylogenies and the comparative method. The American

$555 \quad$ Naturalist 125(1):1-15.

556 Felsenstein, J. (2005). Using the quantitative genetic threshold model for inferences

557 between and within species. Philosophical Transactions of the Royal Society B 
360:1427-1434.

559 FitzJohn, R. G. (2010). Quantitative traits and diversification. Systematic biology, 59(6), $560 \quad 619-633$.

561 Freckleton, R. P., P. H. Harvey, and M. Pagel (2013). Phylogenetic Analysis and

562 Comparative Data. The American Naturalist 160:712-726. doi: 10.1086/343873

563 Fritz, S. A., and A. Purvis (2010). Selectivity in mammalian extinction risk and threat

564 types: A new measure of phylogenetic signal strength in binary traits. Conservation

$565 \quad$ Biology 24:1042-1051. doi: 10.1111/j.1523-1739.2010.01455.x

566 Garamszegi, L. Z., and A. P. Møller (2011). Nonrandom variation in within-species

567 sample size and missing data in phylogenetic comparative studies. Systematic

568 Biology 60:876-880. doi: 10.1093/sysbio/syr060

569 Gaston, K. J. (1994). Rarity. Chapman \& Hall, London.

570 Hansen, T. F. (1997). Stabilizing selection and the comparative analysis of adaptation.

$571 \quad$ Evolution 51:1341-1351.

572 Harvey, M. G., and D. L. Rabosky. (2018). Continuous traits and speciation rates:

$573 \quad$ Alternatives to state-dependent diversification models. Methods in Ecology and

$574 \quad$ Evolution, 9(4), 984-993.

575 Herrera-Alsina, L., and R. Villegas-Patraca (2014). Biologic interactions determining

576 geographic range size: A one species response to phylogenetic community

$577 \quad$ structure. Ecology and Evolution 4:968-976. doi: 10.1002/ece3.959

578 Ingram, T., and D. L. Mahler (2013). SURFACE: Detecting convergent evolution from

579 comparative data by fitting Ornstein-Uhlenbeck models with stepwise Akaike

580 Information Criterion. Methods in Ecology and Evolution 4:416-425. doi: 
582 Jablonski, D. (1987). Heritability at the Species Level: Analysis of Geographic Ranges 583 of Cretaceous Mollusks. Science 238:360-363.

584 Jetz, W., G. H. Thomas, J. B. Joy, K. Hartmann, and A. O. Mooers (2012). The global 585 diversity of birds in space and time. Nature 491:444-8. doi: 10.1038/nature11631 586 Jiang, W., S-Y Chen, H. Wang, D-Z. Li, and J. J. Wiens (2014). Should genes with 587 missing data be excluded from phylogenetic analyses? Molecular Phylogenetics $588 \quad$ and Evolution 80:308-318.

589 Kuhn, T. S., A. Mooers, and G. H. Thomas (2011). A simple polytomy resolver for dated 590 phylogenies. Methods in Ecology and Evolution 2:427-436. doi: 10.1111/j.2041$591 \quad$ 210X.2011.00103.x

592 Losos, J. B. (2008). Phylogenetic niche conservatism, phylogenetic signal and the 593 relationship between phylogenetic relatedness and ecological similarity among 594 species. Ecology Letters 11:995-1003. doi: 10.1111/j.1461-0248.2008.01229.x

595 Louca, S., and M. Doebeli. (2017). Efficient comparative phylogenetics on large trees. 596 Bioinformatics, 34(6), 1053-1055.

597 Malaney, J. L., and J. A. Cook (2018). A perfect storm for mammalogy: declining 598 sample availability in a period of rapid environmental degradation. Journal of 599 Mammalogy 99:773-788. doi: 10.1093/jmammal/gyy082

600 Maurer, B. A. (1991). Concluding remarks: Birds, body size and evolution. In: Acta XX 601 Congressus Internationalis Ornithologici. New Zealand Ornithological Congress 602 Board, Wellington.

603 O’Meara, B. C., C. Ané, M. J. Sanderson, and P. C. Wainwright (2006). Testing for 
604 different rates of continuous trait evolution using likelihood. Evolution; international 605 journal of organic evolution 60:922-33. doi: 10.1111/j.0014-3820.2006.tb01171.x 606 O'Meara, B. C., and J. M. Beaulieu (2014). Modelling Stabilizing Selection: The 607 Attraction of Ornstein-Uhlenbeck Models. In Modern Phylogenetic Comparative 608 Methods and Their Application in Evolutionary Biology (L. Z. Garamszegi, Editor). 609 Springer, Berlin, Heidelberg.

610 Orme, D., R. Freckleton, G. Thomas, T. Petzoldt, S. Fritz, N. Isaac and W.Pearse.

611 (2017). The caper package: comparative analysis of phylogenetics and evolution in $612 \mathrm{R}$, version 1.0.1.

613 Paradis, E. and K. Schliep. (2019). ape 5.0: an environment for modern phylogenetics 614 and evolutionary analyses in R. Bioinformatics 35(3):526-528.

615 Pennell, M. W., J. M. Eastman, G. J. Slater, J. W. Brown, J. C. Uyeda, R. G. FitzJohn, 616 M. E. Alfaro, and L. J. Harmon. (2014). geiger v2. 0: an expanded suite of methods 617 for fitting macroevolutionary models to phylogenetic trees. Bioinformatics, 30(15), $618 \quad 2216-2218$.

619 Pennell, M. W., R. G. FitzJohn, and W. K. Cornwell (2016). A simple approach for 620 maximizing the overlap of phylogenetic and comparative data. Methods in Ecology 621 and Evolution 7:751-758. doi: 10.1111/2041-210X.12517

622 Qian, H., and R. E. Ricklefs (2004). Geographical distribution and ecological

623 conservatism of disjunct genera of vascular plants in eastern Asia and eastern

624 North America. Journal of Ecology 92:253-265. doi: 10.1111/j.0022-

$625 \quad 0477.2004 .00868 . x$

626 Rabosky, D. L. (2009). Heritability of extinction rates links diversification patterns in 
molecular phylogenies and fossils. Systematic Biology, 58(6), 629-640.

628 Rabosky, D. L. (2015). No substitute for real data: A cautionary note on the use of

629 phylogenies from birth-death polytomy resolvers for downstream comparative $630 \quad$ analyses. Evolution 69:3207-3216. doi: 10.1111/evo.12817

631 Reddy, S. (2014). What's missing from avian global diversification analyses? Molecular 632 Phylogenetics and Evolution 77:159-165. doi: 10.1016/j.ympev.2014.04.023

633 Slater, G. J., L. J. Harmon, D. Wegmann, P. Joyce, L. J. Revell,and M. E. Alfaro.

634 (2012a). Fitting models of continuous trait evolution to incompletely sampled 635 comparative data using approximate Bayesian computation. Evolution66-3: 752636762.

637 Slater, G. J., L. J. Harmon, and M. E. Alfaro. (2012b). Integrating fossils with molecular 638 phylogenies improves inference of trait evolution. Evolution 66(12), 3931-3944.

639 Stadler, T. (2010). Sampling-through-time in birth-death trees. Journal of Theoretical $640 \quad$ Biology 267:396-404.

641 Stadler, T., A. Gavryushkina, R. C. Warnock, A. J. Drummond, and T. A. Heath. (2018). 642 The fossilized birth-death model for the analysis of stratigraphic range data under 643 different speciation modes. Journal of Theoretical Biology, 447, 41-55.

644 Thomas, G. H., K. Hartmann, W. Jetz, J. B. Joy, A. Mimoto, and A. O. Mooers (2013).

645 PASTIS: An R package to facilitate phylogenetic assembly with soft taxonomic 646 inferences. Methods in Ecology and Evolution 4:1011-1017. doi: 10.1111/2041$647 \quad 210 \times .12117$

648 Thomson, R. C., and H. B. Shaffer (2010). Rapid progress on the vertebrate tree of life. $649 \quad$ BMC Evolutionary Biology 8:19. 
650 Vamosi, J. C., and J. R. Wilson. (2008). Nonrandom extinction leads to elevated loss of 651 angiosperm evolutionary history. Ecology Letters, 11(10), 1047-1053.

652 Waldron, A. (2007). Null Models of Geographic Range Size Evolution Reaffirm Its 653 Heritability. The American Naturalist 170:221-231. doi: 10.1086/518963

654 Wiens, J. J., D. D. Ackerly, A. P. Allen, B. L. Anacker, L. B. Buckley, H. V. Cornell, E. I. 655 Damschen, T. Jonathan Davies, J. A. Grytnes, S. P. Harrison, B. A. Hawkins, R. D. 656 Holt, C. M McCain, and P. R. Stephens. (2010). Niche conservatism as an 657 emerging principle in ecology and conservation biology. Ecology Letters 13:1310$658 \quad$ 1324. doi: 10.1111/j.1461-0248.2010.01515.x

659 Wiens, J. J., and C. H. Graham (2005). Niche Conservatism: Integrating Evolution, 660 Ecology, and Conservation Biology. Annual Review of Ecology, Evolution, and 661 Systematics 36:519-539. doi: 10.1146/annurev.ecolsys.36.102803.095431

662 Wiens, J. J., and M. C. Morrill (2011). Missing Data in Phylogenetic Analysis: Reconciling 663 Results from Simulations and Empirical Data. Systematic Biology 60(5):719-731. 664 Zuk, M., and M. Travisano (2018). Models on the Runway: How Do We Make Replicas 665 of the World? The American Naturalist 192:1-9. doi: 10.1086/697508

666 667 


\section{Table $\mathbf{1}$ (on next page)}

Summary of simulated traits and their generating models. 
1 Table 1. Summary of simulated traits and their generating models.

\begin{tabular}{|c|c|c|}
\hline $\begin{array}{c}\text { Abbreviation of } \\
\text { trait }\end{array}$ & Description & Model \\
\hline $\mathbf{R}$ & $\begin{array}{l}\text { Binary trait representing } \\
\text { regimes required for BMS } \\
\text { and OUM models for } T\end{array}$ & $\begin{array}{c}\text { Equal-rates MK } \\
\text { model }\end{array}$ \\
\hline$T$ & $\begin{array}{c}\text { Continuous trait about } \\
\text { whose evolution we are } \\
\text { interested in making } \\
\text { inferences }\end{array}$ & $\begin{array}{c}\text { BM, BMS, OU or } \\
\text { OUM }\end{array}$ \\
\hline$S$ & $\begin{array}{l}\text { Binary trait determining } \\
\text { the sampling status of } \\
\text { each tip }(0=\text { missing, } \\
1=\text { sampled })\end{array}$ & $\begin{array}{l}\text { Threshold model } \\
\text { for cluMT; non- } \\
\text { phylogenetic for } \\
\text { corMT }\end{array}$ \\
\hline $\mathbf{L}$ & $\begin{array}{l}\text { Continuous trait } \\
\text { representing the liability } \\
\text { underlying } \mathbf{S} \text { in the } \\
\text { threshold model in the } \\
\text { cluMT scenario }\end{array}$ & BM \\
\hline
\end{tabular}

2 


\section{Table 2 (on next page)}

Model selection error rate.

Number of simulations, for each combination of type of missing taxa, percentage of missing taxa, and generating model for the trait of interest $T$, in which the model with the greatest support (lowest AICC) was not the generating model. The numbers are always out of 1000 replicated simulations. 
1 Table 2. Model selection error rates. Number of simulations, for each combination of

2 type of missing taxa, percentage of missing taxa, and generating model for the trait of

3 interest $\mathrm{T}$, in which the model with the greatest support (lowest AICc) was not the

4 generating model. The numbers are always out of 1000 replicated simulations.

\begin{tabular}{|c|c|r|r|r|r|}
\hline & & BM & BMS & OU & OUM \\
\hline No missing taxa & & 306 & 61 & 181 & 22 \\
\hline Random missing taxa & $10 \%$ & 296 & 53 & 176 & 13 \\
\cline { 2 - 7 } & $50 \%$ & 298 & 70 & 161 & 11 \\
\cline { 2 - 6 } & $90 \%$ & 266 & 83 & 196 & 4 \\
\hline Clumped missing taxa & $10 \%$ & 279 & 63 & 167 & 18 \\
\cline { 2 - 7 } & $50 \%$ & 291 & 63 & 195 & 22 \\
\cline { 2 - 6 } & $90 \%$ & 318 & 60 & 194 & 24 \\
\hline Correlated missing & $10 \%$ & 300 & 47 & 178 & 25 \\
\cline { 2 - 6 } taxa & $50 \%$ & 305 & 73 & 177 & 16 \\
\cline { 2 - 6 } & $90 \%$ & 381 & 99 & 169 & 11 \\
\hline
\end{tabular}

5 


\section{Table 3 (on next page)}

Delta AICc of the generating model when it was not selected as the top model.

Median delta AICC of the generating model for the trait of interest $\mathbf{T}$ in the set of simulations, under each combination of type of missing taxa, percentage of missing taxa and generating model, in which the generating model was not the model with the greatest support. 
1 Table 3. Median delta AICc of the generating model for the trait of interest $\mathrm{T}$ in the set of

2 simulations, under each combination of type of missing taxa, percentage of missing taxa

3 and generating model, in which the trait of interest was not the model with the greatest

4 support (lowest AICc).

\begin{tabular}{|c|c|c|c|c|c|}
\hline & & BM & BMS & $\mathrm{OU}$ & OUM \\
\hline No missing taxa & & 1.592 & 2.127 & 1.422 & 1.791 \\
\hline \multirow[t]{3}{*}{ Random missing taxa } & $10 \%$ & 1.676 & 1.471 & 1.215 & 1.826 \\
\hline & $50 \%$ & 1.759 & 1.927 & 1.459 & 25.669 \\
\hline & $90 \%$ & 1.577 & 1.508 & 1.391 & 4.261 \\
\hline \multirow[t]{3}{*}{ Clumped missing taxa } & $10 \%$ & 1.966 & 1.784 & 1.047 & 1.370 \\
\hline & $50 \%$ & 1.866 & 2.174 & 1.196 & 2.325 \\
\hline & $90 \%$ & 1.811 & 2.079 & 1.210 & 1.515 \\
\hline \multirow{3}{*}{$\begin{array}{l}\text { Correlated missing } \\
\text { taxa }\end{array}$} & $10 \%$ & 1.865 & 2.364 & 1.414 & 1.364 \\
\hline & $50 \%$ & 1.621 & 1.442 & 1.243 & 3.016 \\
\hline & $90 \%$ & 2.252 & 2.353 & 1.441 & 3.875 \\
\hline
\end{tabular}

5 


\section{Table 4 (on next page)}

Bias of parameter estimates

Bias of parameter estimates from models fitted to data simulated under various scenarios and proportions of missing taxa. Bias was calculated as the generating parameter value, minus the mean estimated parameter across 1000 simulations, divided by the generating parameter value. Types of missing taxa: nMT: no missing taxa; rMT: random missing taxa; cluMT: phylogenetically clumped missing taxa; corrMT missing taxa correlated to the trait of interest). 


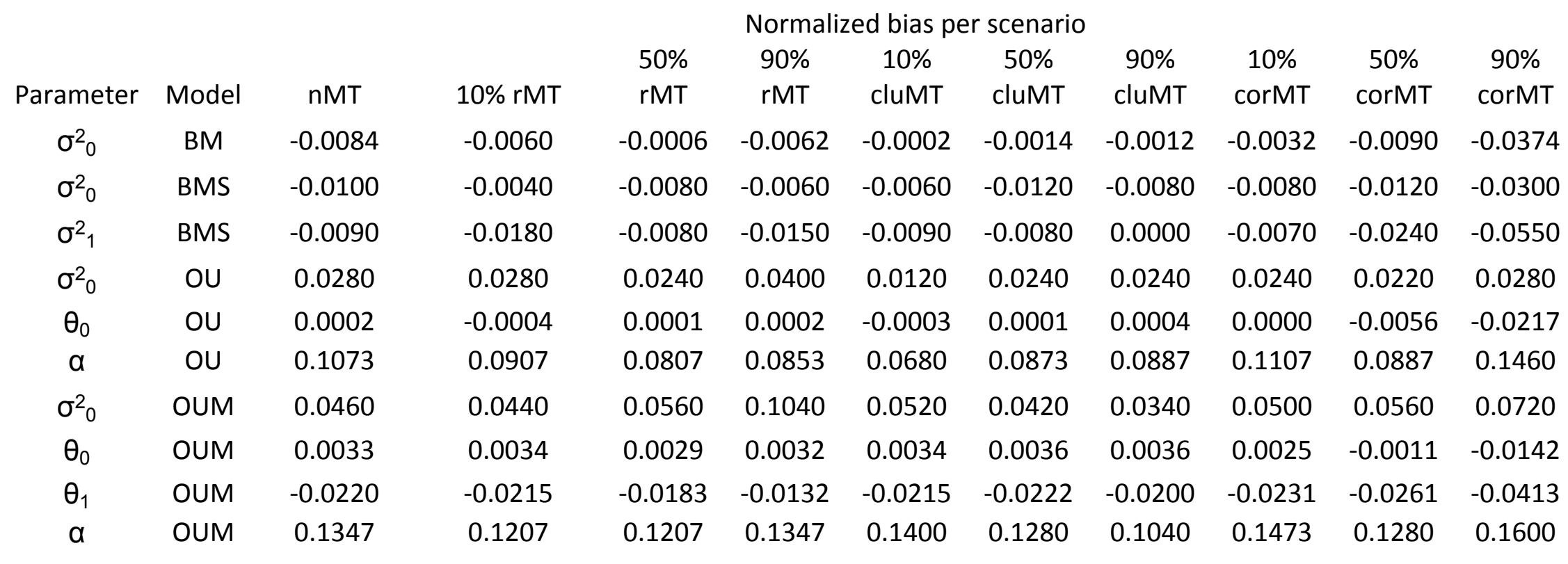




\section{Table 5 (on next page)}

Precision of parameter estimates

Precision of parameter estimates from models fitted to data simulated under various scenarios and proportions of missing taxa. Precision was calculated as the median deviation from the median estimated parameter across 1000 simulations, divided by the generating parameter value. Types of missing taxa: nMT: no missing taxa; rMT: random missing taxa; cluMT: phylogenetically clumped missing taxa; corrMT missing taxa correlated to the trait of interest). 
1

\begin{tabular}{|c|c|c|c|c|c|c|c|c|c|c|c|}
\hline \multirow[b]{2}{*}{ Parameter } & \multirow[b]{2}{*}{ Model } & \multicolumn{10}{|c|}{ Normalized precision per scenario } \\
\hline & & nMT & $10 \%$ rMT & $50 \%$ rMT & $90 \%$ rMT & $10 \%$ cluMT & $50 \%$ cluMT & $90 \%$ cluMT & $10 \%$ corMT & $50 \%$ corMT & $90 \%$ corMT \\
\hline$\sigma_{0}^{2}$ & BM & 0.0800 & 0.0768 & 0.0838 & 0.0782 & 0.0792 & 0.0816 & 0.0810 & 0.0862 & 0.0756 & 0.0834 \\
\hline$\sigma_{0}^{2}$ & BMS & 0.1080 & 0.1000 & 0.1040 & 0.1040 & 0.1080 & 0.1000 & 0.1120 & 0.1020 & 0.1020 & 0.1020 \\
\hline$\sigma^{2}{ }_{1}$ & BMS & 0.1340 & 0.1380 & 0.1420 & 0.1440 & 0.1430 & 0.1420 & 0.1400 & 0.1350 & 0.1460 & 0.1460 \\
\hline$\sigma_{0}^{2}$ & OU & 0.1180 & 0.1180 & 0.1160 & 0.1500 & 0.1240 & 0.1140 & 0.1140 & 0.1220 & 0.1220 & 0.1460 \\
\hline$\theta_{0}$ & $\mathrm{OU}$ & 0.0090 & 0.0084 & 0.0079 & 0.0068 & 0.0085 & 0.0090 & 0.0088 & 0.0082 & 0.0082 & 0.0071 \\
\hline$\alpha$ & OU & 0.2893 & 0.2587 & 0.2727 & 0.2520 & 0.2840 & 0.2673 & 0.2580 & 0.2673 & 0.2567 & 0.2547 \\
\hline$\sigma_{0}^{2}$ & OUM & 0.1260 & 0.1220 & 0.1360 & 0.1660 & 0.1160 & 0.1200 & 0.1180 & 0.1300 & 0.1260 & 0.1580 \\
\hline$\theta_{0}$ & OUM & 0.0106 & 0.0090 & 0.0089 & 0.0077 & 0.0102 & 0.0098 & 0.0094 & 0.0090 & 0.0089 & 0.0078 \\
\hline$\theta_{1}$ & OUM & 0.0141 & 0.0145 & 0.0147 & 0.0144 & 0.0141 & 0.0149 & 0.0160 & 0.0135 & 0.0134 & 0.0108 \\
\hline$\alpha$ & OUM & 0.2893 & 0.2760 & 0.2727 & 0.2847 & 0.2813 & 0.2787 & 0.3027 & 0.3147 & 0.2953 & 0.2873 \\
\hline
\end{tabular}




\section{Figure 1}

Illustration of the various simulated configurations of missing taxa.

(A-C) Trees depicting a trait $\mathbf{T}$ simulated under an OUM model, and random (A), phylogenetically clumped (B), or correlated (C) missing taxa. (D) Selective regimes, simulated under an MK model, underlying variation in the theta parameter in the OUM model used to simulate $\mathbf{T}$ in A-C. The tree in this figure contains 333 tips, 33 of which were simulated to be missing in each panel, thus corresponding to a $10 \%$ missing taxa scenario. 

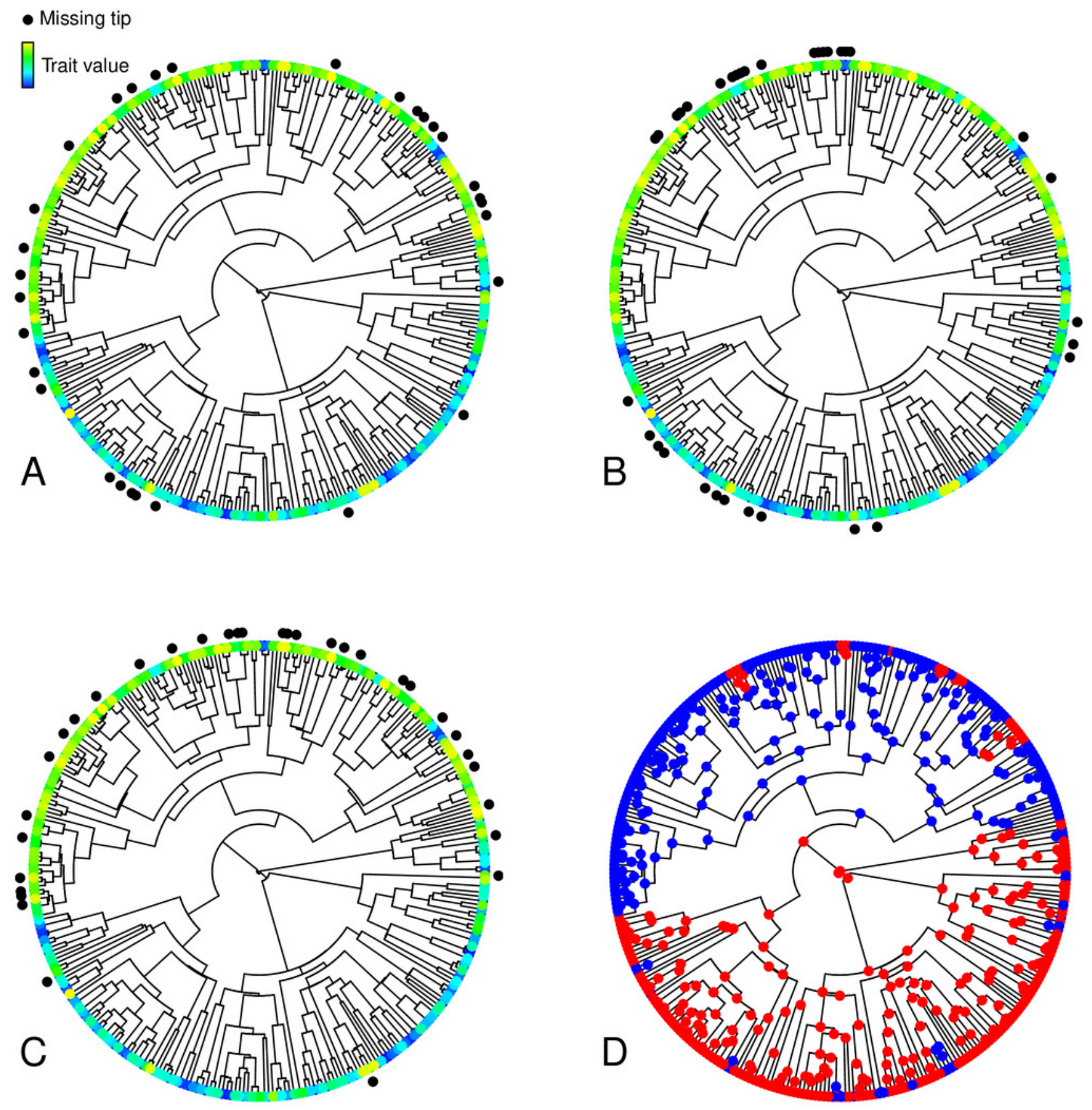


\section{Figure 2}

\section{Best-fitting models selected in each set of simulations}

Bar plots depicting the number of simulations in which each model was selected by AICc as the best fitting model, under each combination of type of missing taxa, percentage of missing taxa, and trait of interest generating model. Types of missing taxa: nMT: no missing taxa; rMT: random missing taxa; cluMT: phylogenetically clumped missing taxa; corrMT missing taxa correlated to the trait of interest) 


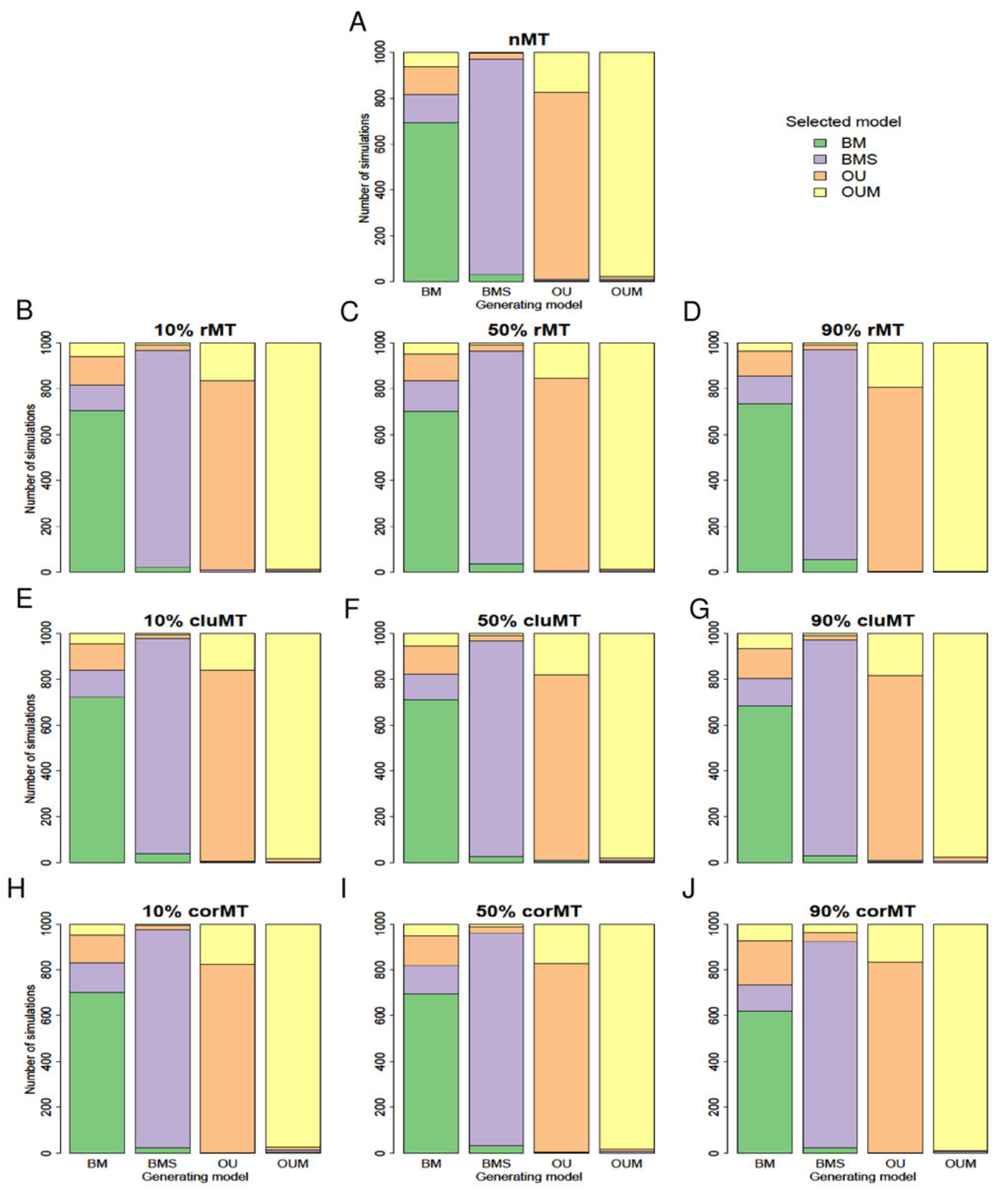




\section{Figure 3}

\section{Distribution of parameter estimates}

Distribution of estimated parameter values for each parameter in each combination of type of missing taxa, percentage of missing taxa and trait of interest generating model. The dotted lines represent the real, generating parameter values. The type and percentage of missing taxa are indicated at the bottom of the figure, whereas the model and parameters are shown at the right-hand edge of the figure.Types of missing taxa: nMT: no missing taxa; rMT: random missing taxa; cluMT: phylogenetically clumped missing taxa; corrMT missing taxa correlated to the trait of interest) 

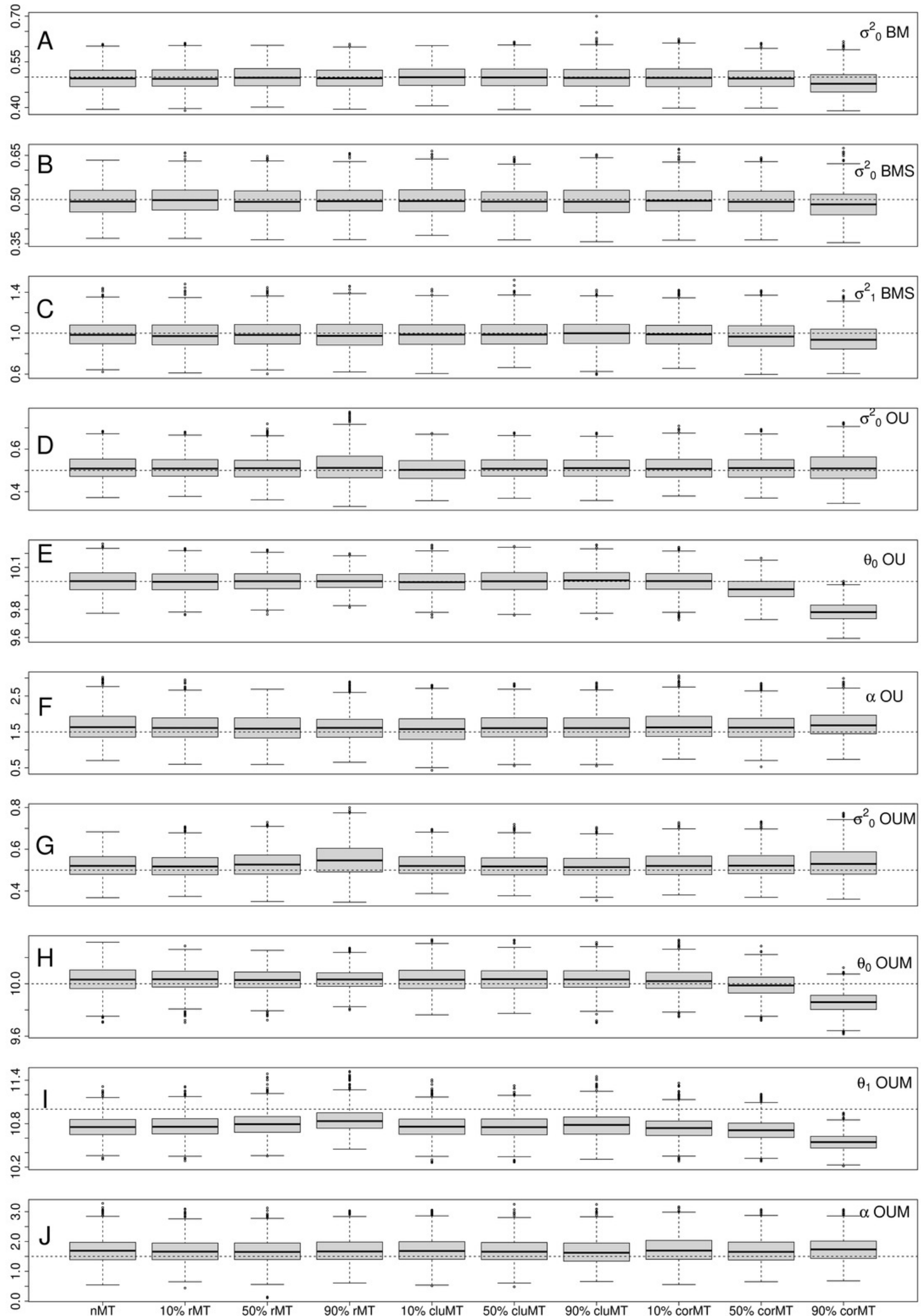